\title{
Filigrane
}

Écoutes psychothérapiques

\section{Discours analytiques et logiques institutionnelles. Entre applications théoriques et implication clinique}

\section{Jacques Cabassut et Mohammed Ham}

Volume 17, numéro 1, printemps 2008

L’avenir du clinicien I

URI : https://id.erudit.org/iderudit/018796ar

DOI : https://doi.org/10.7202/018796ar

Aller au sommaire du numéro

Éditeur(s)

Revue Santé mentale au Québec

ISSN

1192-1412 (imprimé)

1911-4656 (numérique)

Découvrir la revue

Citer cet article

Cabassut, J. \& Ham, M. (2008). Discours analytiques et logiques institutionnelles. Entre applications théoriques et implication clinique.

Filigrane, 17(1), 165-180. https://doi.org/10.7202/018796ar

\section{Résumé de l'article}

Le sujet freudien comme la clinique de transfert sont sûrement remis en cause aujourd'hui par les nouveaux modes de gouvernance, dispositifs ou les différents savoirs « scientistes » qui viennent encombrer le clinicien au quotidien. Cependant, à l'heure où ce dernier est de plus en plus missionné pour une pratique non conventionnelle, et d'autant plus s'il se réfère à l'éthique analytique, comment peut-il éviter de produire de l'exclusion par gommage de toute altérité sémantique au sein même des interventions, des actes de paroles et des échanges langagiers qui sont les siens ? C'est ce risque, accru dans la clinique contemporaine et le lien de transfert, que les auteurs ont mis à l'épreuve de leur pratique et ce, afin de distinguer la psychanalyse appliquée d'une clinique impliquée. 


\title{
Discours analytiques et logiques institutionnelles. Entre applications théoriques et implication clinique
}

\author{
jacques cabassut \\ mohammed ham
}

\begin{abstract}
Le sujet freudien comme la clinique de transfert sont sûrement remis en cause aujourd'hui par les nouveaux modes de gouvernance, dispositifs ou les différents savoirs «scientistes» qui viennent encombrer le clinicien au quotidien. Cependant, à l'heure où ce dernier est de plus en plus missionné pour une pratique non conventionnelle, et d'autant plus s'il se réfère à l'éthique analytique, comment peut-il éviter de produire de l'exclusion par gommage de toute altérité sémantique au sein même des interventions, des actes de paroles et des échanges langagiers qui sont les siens? C'est ce risque, accru dans la clinique contemporaine et le lien de transfert, que les auteurs ont mis à l'épreuve de leur pratique et ce, afin de distinguer la psychanalyse appliquée d'une clinique impliquée.
\end{abstract}

\section{Liminaire}

a démarche «soin de qualité » dans le domaine de la santé... La loi nouvelle du 2 janvier 2002 qui régit à présent en France le secteur du médico-social... L'invasion du «discours de la science», à partir de modèles épistémologiques «durs», sur ces lieux de production et de transmission de savoirs que sont les universités...

Autant de points qui illustrent la fragilité contemporaine du sujet freudien sur les scènes praxiques, heuristiques et institutionnelles de la clinique analytique. En tant que cliniciens praticiens (en institution du social, du médico-social), mais aussi analystes en cabinet, superviseurs et enseignants-chercheurs d'une université française, nous sommes donc partagés quotidiennement entre ces nouvelles formes de gouvernance et une écoute des phénomènes de l'inconscient, confrontés aux logiques de management d'équipes comme aux effets de la pulsion de mort dans ces dernières, engagés dans une éthique de notre mission universitaire de formation des futurs psychologues cliniciens mais également soumis à la pression administrative quant à la professionnalisation rapide de bataillons de psychothérapeutes ${ }^{1} \ldots$

Nous avons pu observer à ce titre, tant sur le terrain que dans la théorie analytique, cette crispation défensive sur un discours de savoirs psys dont la compilation à d'autres approches - il faut bien de la pluridisciplinarité ! — ou bien l'isolement voire la fétichisation d'une clinique psychanalytique - qui ne se 
supporte que d'une pure majuscule — dans les différents dispositifs d'enseignements, de pratiques, et de recherche, versent dans l'application au détriment de l'implication clinique. Au risque bien entendu, de corrompre les prises en charge comme les dispositifs expérimentaux, toutes ces nouvelles zones d'exercice clinique (sleeping, MJC, banlieues dites difficiles, maison d'arrêt...) qu'ils sont censés promouvoir. Il va sans dire que ces pratiques professionnelles novatrices se construisent aujourd'hui, entre autres à l'université, avant de se constituer en avenir du psychologue clinicien...

L'exclusive de tels discours, «imaginarisés » dans leur aspect défensif plutôt que ré-créatif, ne viendrait-elle pas en miroir, sur un mode contre-transférentiel, traduire — c'est-à-dire révéler tout en trahissant — les problématiques d'exclusion $^{2}$ ? Ces dernières n'éprouvent-elles pas suffisamment le «psy », pour le déporter dans un enfermement sémantique qui viendrait l'exclure de divers champs d'intervention possibles?

Telle est l'hypothèse que nous allons mettre en travail ici, afin de mieux cerner cette heuristique analytique qui nous invite à une connaissance de l'objet en tant que malaise inter et intra subjectif. Notre entreprise visera à démontrer que, quel que soit l'espace socio-professionnel dans lequel se déploient nos pratiques, il n'existe pas d'autre lieu d'exercice clinique que celui de notre propre cadre psychique.

\section{Massification identitaire : séquence...}

« Je suis un éducateur spécialisé et je ne vois pas pourquoi mon travail doit être contrôlé par un psychanalyste!» s'écria, à la face de son collègue psychologue clinicien, cet intervenant en toxicomanie à bout d'arguments. Ce dernier lui rétorqua: «Tu t'occupes de toxicomanes et à ce que je sache, tu n'en es pas un », ce qui fit rire le reste de l'équipe dite pluridisciplinaire, composée d'une douzaine de personnes, et amena le psychanalyste superviseur à proposer la pause, habituelle, une vingtaine de minutes plus tôt qu'à l'accoutumée. En fait, lors de cette première séquence de contrôle, une joute oratoire à trois s'était installée entre le psychologue, l'éducateur et l'infirmière. L'atmosphère des échanges était assez tendue, voire hostile, due en apparence à des divergences de points de vue concernant la prise en charge d'un patient désigné toxicomane. Les propos furent assez discourtois et âpres, chacun tentant de légitimer son intervention par une remise en question implicite ou explicite de l'intervention de l'autre.

\section{Géographies du mot}

Ainsi, seul le statut professionnel sert de tenant lieu qui autorise, dans le langage, la mise en scène d'un savoir vide qui offre au corps professionnel de chacun, l'illusion de sa pseudo identité professionnelle («Je suis éducateur, psychologue, infirmière, assistante sociale, etc.»). Chacun se fond alors dans un « on» symbiotique auto-désubjectivant, aliéné à l'ordre et à l'opacité d'un code singulièrement commun. Conjointement, celui-ci génère un savoir sur l'entité, 
l'espace d'où procède le discours d'autrui, ce qui met en marche la machine à exclure, également procédé de désubjectivation de l'autre.

Cette exclusion dans et par le langage, alors captive d'un discours limitant ses prétentions à un «géographisme» corporatiste et professionnel, n'en demeure pas moins éclairante quant à son but, son objet et sa source, d'un point de vue pulsionnel trans et inter subjectif.

a) Pour ce qui concerne son but, il milite, entre autres, en faveur d'une satisfaction imaginaire qui tend à mettre le semblable à l'endroit de l'identique, afin de continuer de s'auto-gérer via le conventionnel, de naviguer dans le connu, d'éviter que le familier ne soit rendu à son état naturel d'étranger. Bref, l'imaginaire de la fonction, du statut, de l'appartenance se substituent au symbolique de la place. S'ouvrir à «l'allogène» qui est en nous, c'est prendre le risque, en lieu et place d'un "géographisme» professionnel, communautaire, identitaire, frontalier, etc... de s'engager dans les voies tumultueuses et sinueuses d'un «géographisme du signifiant» (Pujol, 1992), seul à même de dés-enkyster la fixité d'un mot (éducateur, psychologue, assistante sociale, toxicomane, français, arabe...) arrêté, entendu et donc constitutif de territoires dans les situations d'interlocutions.

b) L'objet, quant à lui, se confond avec le sujet du discours. Il mobilise à ce titre, dans l'échange langagier, un investissement d'objet (soit ce qui est posé devant le sujet) qui incite la pulsion à l'investir - frontalement - de manière amoureuse, haineuse ou pire, abjecte. Dans la séquence évoquée, elle réactive chez l'éducateur une angoisse sensitive qui oblige celui-ci à sortir du champ du regard — ciblé — de l'A(a)utre : pourquoi devrait-il être super-visé par un psychanalyste? Elle conduit également le psychologue au rejet des interventions du dit éducateur, interventions qui, comme il le formule, le perturbe, attribuant à ce dernier une autre place, celle de «nontoxicomane» par le truchement d'une relation transférentielle dont le patient se plaindrait. Enfin, elle plonge l'infirmière dans l'incompréhension et le désaccord avec ses collègues, car elle reste, quant à elle, satisfaite des progrès réalisés par le patient depuis que celui-ci est «substitué»; si ce dernier ne respecte pas toujours ses rendez-vous avec le psychologue, pour «la Metha $^{3} »$ il est à l'heure.

Bref, ces différentes séquences nous instruisent de l'effet de glissement du symbolique (des places) à l'imaginaire ${ }^{4}$ (de la fonction), qui (dé) limite la géographie institutionnelle, conceptuelle, représentationnelle, administrative de chacun. $\mathrm{L}^{\prime}$ «On» ne peut se penser qu'à partir du lieu de son exclusion, qui est aussi et surtout celle l'A(a)utre. Confusion de lieux d'où «ça» parle donc: celui du supposé savoir la vérité, à celui d'un docte sujet sachant. Assimilation du signifiant au signe ensuite: celui-ci dans sa fonction de «représenter quelque chose pour quelqu'un ${ }^{5} »$, peut se constituer en objet narcissique, source de conflit de personnes, et ce, au risque d'une inflation de «jargonophasie», qui fera office de lien - c'est à dire à la fois ce qui lie et sépare de l'autre. L'amour et la haine ${ }^{6}$ 
lient et séparent de l'A(a)utre... sans pour autant résoudre la division structurelle, intime, endogène qui le touche en tant qu'animal parlant.

Nous avançons que pour ne pas exclure l'autre («Je» est un «A(a)utre») du discours et donc du réel de sa pratique, toute interprétation se devrait d'être, d'abord, délivrée pour soi. Elle prendrait alors un caractère extime ${ }^{7}$ (Soi provient de l'Autre) et convoquerait le sujet à sa condition d'étranger au et de la langue. À la familiarité des échanges — l'on se comprend — se substituerait l'étrangeté sinon comprise tout au moins entendue — de sa condition de parlêtre.

En effet, pour la psychanalyse et elle seule, ce qui la démarque de n'importe quelle autre approche psychologique voire psychologisante, le sujet pour s'adresser à l'autre semblable, l'autre de la réalité, l'interlocuteur dans l'échange, en passe d'abord par l'Autre ${ }^{8}$. Chacun de nous parle toujours et d'abord à cet Autre, intime -c'est pourquoi «je» peux me parler à moi-même. Cet autre du discours supporté le plus souvent par un quidam «[...] reste toujours dans le fond de la parole du locuteur un autre construit par lui, un autre non séparé ». (Lesourd, 2006, 103). Autrement dit, dans l'échange langagier inter humain, chaque petit autre peut potentiellement incarner l'Autre pour le sujet.

\section{Exclusion logique et logiques de l'exclusion}

Cet A(a)utre, est également un Autre institutionnel, au sein duquel se déploie notre exemple d'échanges langagiers. En effet, "l'institution logiquement première, c'est la langue» (Legendre, 2004, 91).

Ainsi pouvons-nous considérer toute institution, comme lien, lien social, lien langagier, en tant qu'espace d'interprétation, bref un espace psychique où se jouent et se déploient des rencontres et des affrontements entre plusieurs versions de l'humain. Les conflits et les malaises qui s'y déroulent, pourront y être entendus à partir du paradigme de l'exclusion dans le langage. La séquence de supervision suscitée, insiste sur cet état de fait. L'exclusion est institutionnalisée; le professionnel qui exerce dans le dit espace, s'apparente et est apparenté à une entité qui tend à le différencier collectivement — parfois dans un réflexe communautaire voire corporatiste —, et non singulièrement de l'autre.

$\mathrm{Au}$ sein de nos institutions, notre travail de clinicien consisterait à être à l'écoute et à accompagner des malaises qui ne sont souvent, selon le canevas proposé ci-dessus, qu'une conjugaison entre deux processus, l'abolition de la singularité et l'objectivation de l'être. Fonctionnement causaliste qui amène l'extérieur, l'autre, à ne plus être qu'une nouvelle catégorie persécutrice.

Hélas, les psychologues cliniciens eux-mêmes, pourraient être amenés à verser dans une exclusive de l'altérité, au lieu d'interroger, d'accompagner ces malaises par une écoute. L'affrontement évoqué en est une illustration: plutôt que de faire fonctionner l'hypothèse de l'inconscient (Freud, 1915, 66), il nous semble que le psychologue tendrait à défendre ce concept comme une entité objectivable et localisable qui envahit l'écoute, l'empêchant de montrer et prenant le sujet au mot, un mot qui prend alors valeur de chose. Le jugement freudien d'attribution et celui 
d'existence entrent ici dans une confusion sémantique, qui s'avère anéantissement du symbolique.

L'idéologisation de la découverte freudienne s'avère, en effet, antipsychanalytique. Elle amène la psychologie clinique versus psychanalyse à ne plus pouvoir être opérationnelle du côté des malaises dans le lien social, au profit d'une application de la théorie psychanalytique. Cette dernière incite au mieux disant, plutôt qu'à l'implication. Elle laisse peu de place à la découverte dans l'éprouvé, à la connaissance (mathos) acquise par la souffrance (pathos), à ce savoir dans l'épreuve cher à P. Fédida $(1992,23)$. Celle-ci, pour ce qui la concerne, n'a de cesse de déconstruire ses interprétations, qu'elles qu'en soient leurs sources théoriques, pour tenter d'apprendre du mouvement discursif et de l'opérationnalité dynamique du dit. Ainsi, le pathéi mathos confronte-t-il le sujet au risque du maldisant, obligeant chacun à se rapprocher du reste de la langue et de la finitude du parlêtre, celle-là même qui convoque son point de butée du réel, son impossible à dire, et offre au clinicien une traversée de sa souffrance via le malaise ambiant.

Il ne s'agirait pas tant de céder sur l'hypothèse de l'inconscient ou les autres concepts que nous avons pris l'habitude de qualifier de fondamentaux, que de dialectiser nos convictions théoriques par les dimensions d'Altérité, qui seront alors incarnées par tous ces petits autres qui ne partagent pas la langue «hypothétique» de l'inconscient. Comment l'éducateur, l'infirmier, l'assistante sociale, l'animateur de quartier, l'instituteur dans une banlieue réputée difficile, le médiateur etc. peuventils encore assumer leur mission au sein d'une démarche d'aide et d'accompagnement moral ou physique? Comment soutenir le besoin du précaire, de l'exclu, du S.D.F, du rmiste, du délinquant, du toxicomane etc. ? Comment se repérer dans des situations de maltraitances, de violences conjugales ou familiales, de demandes d'aides matérielles, si les sollicitations ne sont plus alors entendues que dans leur dimension désirante, sans prendre en compte la question des nécessités de survie?

Autrement dit, doivent-ils se cantonner à cette supposée hauteur symbolique, alors que leurs pratiques présupposent souvent du faire imaginaire, voire de l'agir avec l'autre, dans le réel de sa condition et de sa souffrance?

\section{D’un symbolisme idéologique aux nécessités du symbolique}

«Fourguer» ses positions à l'autre au nom d'un idéalisme symbolique, est un «idéologisme» qui ignore que, topiquement, le symbolique ne peut se soutenir que des deux autres dimensions constitutives de l'assise du sujet, à savoir celle du réel et de l'imaginaire. À méconnaître et/ou à bannir systématiquement la dimension imaginaire, sans l'inscrire dans une temporalité logique ${ }^{9}$ qui lui laisse la possibilité de s'instruire de sa part symbolique, le clinicien ne court-il pas le risque d'une intervention/effraction qui confronte l'autre à du réel innommable, l'acculant dans une position de plaintes, d'agressivité et de dépressivité tel le sujet dit «névrosé traumatique»? Il ne resterait alors à ce dernier, pour se soutenir, que le souvenir événementiel qui ne cesserait d'insister comme ligature, cadenas qui la lui boucle, qui enferme le sujet au dehors, telle une interprétation sauvage ${ }^{10}$. 
Les psychologues cliniciens ont-ils à exercer leur métier dans ces nouveaux champs ${ }^{11}$ ? Ont-ils à travailler aux côtés des acteurs sociaux précédemment évoqués, et à les soutenir dans leur pratique ? Dans les deux cas, nous répondrons par l'affirmative, à condition qu'ils ne cèdent pas sur ce qui relève du symbolique du désir et de l'éthique. Pour ce faire, nous proposerons quelques pistes de réflexions cliniques et métapsychologiques visant donc à :

- échapper à la diabolisation de la dimension imaginaire ;

- éviter la confusion entre justesse des convictions et vérité de savoirs ;

- récuser l'idéalisation, voire d'idéologisation des notions cliniques analytiques ;

- refuser tout militantisme narcissique à visage clinico-psychologique, ou psychanalytique, afin de ne pas réduire l'éthique de la psychanalyse ou de la psychologie clinique à une croyance.

Il s'agirait plutôt de déployer une heuristique au sein d'une démarche qui tiendrait compte des malaises inter subjectifs qui sont en affrontement dans ces lieux. Cette démarche ne supposerait pas seulement une écoute dite «bienveillante », mais nécessiterait une implication. Elan processuel qui s'attache à montrer, plutôt qu'à toujours dire. Elle serait ainsi animée par une impulsion éthique vers l'autre, confrontation où la relation en gestation ne cesse de nous instruire, via l'analyse et l'hostilité des enjeux transféro-transférentiels. Cette implication consisterait à se déprendre, constamment, de son savoir, afin que l'autre puisse apprendre de sa propre subjectivation dans le dire ou le tu. Par ce biais, c'est un imaginaire non redoutable qui peut se soutenir, cette fois dans ses capacités créatrices, dans la mesure où il sera décontaminé — sinon purgé — de sa haine spéculaire. Le besoin est alors entendu et susceptible d'être accompagné pour éventuellement ouvrir vers le désir, afin que la pratique dans les champs de l'exclusion ne se transforme pas en exercice d'exclusion.

\section{Le sentiment d'appartenance ou la part tenace du discours}

L'exclusion générée par un savoir exclusif pousserait le clinicien détenteur à ne pas appliquer à sa propre écoute les concepts dont il se fait la porte parole. Il risque de les réserver aux pratiques des autres, oubliant au passage que l'inconscient est une hypothèse, et que sa validité réside dans une dimension qui laisse ouverte à chaque rencontre la possibilité de la mettre «à l'épreuve de la parole» (Gori, 1996). Celle-ci ferait alors raisonner chez autrui un message pour le moins paradoxal: «faites ce que je dis» se transformerait en «ne faites pas ce que je ne dis pas mais que vous entendez».

L'idéologisation à outrance de toute métapsychologie, ou courant théorique, dont pourrait se prévaloir le clinicien en termes d'appartenances lexicales, d'écoles, de sociétés, d'associations, l'amènerait à une position où le savoir filtre la parole pour se retrouver dans des jugements à diagnostics qui voilent, autant qu'ils dévoilent, les impasses de leurs procédés. Il s'agirait là, à l'aide du savoir sur l'autre, de se protéger de l'éprouvé transféro-contre-transférentiel qui nous 
traverse, de l'extérieur — par une nosographie, déjà-là — et par le dedans — inquiétante étrangeté, déjà-là également-.

Concernant cette aliénation dans l'appartenance, il est assez remarquable de noter que «apparenté », «appartenance» et «appartement» semblent manifestement se tenir et se soutenir de la même étymologie, que condense le mot dépendance. Ils s'inscrivent dans un mouvement linguistique autant pertinent qu'impertinent, trajet paradoxal qui camoufle, autant qu'il exhibe, un lien de dépendance. Ce dernier n'est «autre» que la subsistance d'un lien fusionnel, un état illusoire d'être, telle cette anticipation imaginaire qui précipite, via l'épreuve de l'identification fondamentale, dans une aliénation nécessairement structurelle, où le «je»si inconsistant soit-il, ne cesse de se déconstruire (Derrida, 1999) via ses références à des appartenances multiples. La question de l'appartenance, qu'elle soit d'ordre culturel, linguistique, religieux, national, de chapelles ou de toutes sortes, semble inscrire un sentiment de nécessité à «référent identitaire». Elle paraît se consigner au cœur de processus d'identifications qui sont à l'œuvre du côté de l'émergence du sujet, comme nous le révèle J. Lacan à travers l'épreuve du miroir, et telle qu'elle ne cesse de se déduire de l'expérience analytique. Elle semble traduire un lien consenti de dépendance et d'allégeance au code de l'autre, lien «miroitier», langagier, virtuel qui se veut consistance identitaire. Celle-ci ne tient que par d'éphémères destinés, marquées du sceau d'interchangeabilités mises au service de dynamiques narcissiques.

La constitution de ce sentiment est en elle-même aliénation, consubstantielle à cette captation par sa propre image, qui refoulée, n'en demeure pas moins de manière dynamique assez tyrannique ${ }^{12}$, fixant l'autre ou les autres dans des schèmes identitaires représentatifs d'inscriptions infantiles de l'Autre.

Le sentiment d'appartenance spécialise l'écoute, cette dimension se redouble souvent par les dispositifs à objets exclusifs de soins, d'accompagnements, d'aides sociales ou administratives, de préventions... dans lesquels les cliniciens sont amenés à exercer leur métier. Ecoutons plutôt la confrontation de l'un de nous, avec un patient rencontré pendant quelques temps dans un centre de soin pour toxicomane.

\section{L'écoute, entre exclusion et spécialisation}

«Les toxicomanes sont anormaux [...]. Quand j'étais toxicomane je prenais de l'héroïne pour être normal». Fragment d'un discours, plus qu'amoureux de son héroïne, d'un patient qui a cessé d'en prendre, mais qui ne cesse d'en causer, pour l'excuser ou la rejeter, la malmener ou la chérir.

Il ne pouvait s'empêcher d'y penser sauf à être dans un activisme à toute épreuve. Son héroïne est apparue une fois de plus, lors de cette rencontre, comme consécutive à une envie «d'en prendre » qu'il avait eue en salle d'attente, après avoir détecté sa présence dans le regard d'un autre patient.

«C'est sûr, il en a pris avant de venir, il était en pleine montée [...]. Comment peuvent-ils venir dans un centre pour se soigner de la drogue et en prendre en même 
temps ?» Mais avant que son «ex-toxicomanie», via l'hérö̈ne, ne soit inscrite dans le registre de la normalité, il y eut un intervalle ou plusieurs actes délictueux et conduites de ces «toxicomanes anormaux» furent détaillés et qualifiés: vols, arnaques, mensonges, malhonnêteté... Les récits ont ainsi cheminé, au gré de souvenirs à caractère événementiel, venant agrémenter, justifier ses jugements.

Notre écoute n'arrivait plus à se déprendre de ses constructions «cinématographiques», renforcées autant par le savoir empirique, que théorique, acquis depuis des années dans ce centre de soins pour «toxicomanes». Loin de se déconstruire, ce savoir s'enflait lors de ces rencontres, nourri par ses récits qui trouvaient dans les repères, imaginairement et défensivement, théoriques le chemin d'y adhérait. D'autant que cette entrevue se situait au décours de vols avec effraction que le centre de soins avait subis, à cette même période, de la part de «toxicomanes », pensait-on.

Par ailleurs, ses propos s'inscrivaient dans une sorte de satisfaction relative que nous éprouvions, du fait de l'abstinence qu'il s'imposait depuis quelques mois. Par moments, nous nous surprenions dans une écoute attentive aux éléments discursifs qui auraient pu en constituer la preuve. Le patient avait-il perçu cette adhésion silencieuse à son discours?

La suite narrative impliqua alors son «je»: «Quand j'étais toxicomane je prenais de l'héroïne pour être normal.» Ces mots tombèrent comme un bruit, un vacarme dans ce colloque à congratulations discursives. Par tous ses récits et toutes ses assertions, le patient clamait sa «normalité toxicomaniaque», en lieu et place de notre satisfaction à ce qu'il ne prenne plus de l'héroïne, nous qui lui «voulions du bien», en substituant notre désir au sien. Là même où nous nous complaisions dans son abstinence, nous incriminions l'hérö̈ne et ne l'entendions que comme produit, et non comme mot dans ses effets subjectifs. Cette héroïne/produit était là, à la base de sa souffrance, de cette vie d'errance, de son quotidien disloqué, de sa marginalité, de ses entrées et sorties de prison, intervalles ponctués de ruptures avec la drogue, de son hépatite $\mathrm{C}$ qui, médicalement depuis l'abstinence et le traitement, semblait régresser.

Pourtant, par la remémoration de sa «normalité toxicomaniaque», le patient venait pointer, dans notre actualité, ce paradigme ou la rupture avec son autrehéroïne ne constituait pas pour autant une séparation.

Il mit en question par là même la manière que nous avions de l'écouter et d'investir la relation. Ne s'était-il pas, à plusieurs reprises, plaint des inquiétudes de sa mère qui ne cessait de le questionner sur la drogue, sur ses allées et venues?

Notre écoute n'agissait-elle pas de la même manière, silencieusement inquiète et protectrice à son égard ? Dès lors le patient ne prit plus d'hérö̈ne, mais revendiqua sa «normalité toxicomaniaque».

En procédant ainsi, notre écoute avait suspendu toute possibilité de se déloger de l'événementiel autant social que médical. Nos rencontres, dans le foyer du langage, étaient assiégées par cette quête d'indices afin d'alimenter la satisfaction, un ravissement qui avait pour fonction de cadenasser les mots et d'éviter toute 
prise de risque par la parole, là où naguère le patient ne cessait de prendre des risques au travers «d'agirs » qui le conduisaient à l'hôpital ou en maison d'arrêt. Notre écoute s'était immobilisée dans une norme qui incriminait l'héroïne comme produit néfaste et dangereux, elle se faisait greffe d'une loi juridique ${ }^{13}$ qui assimile le toxicomane à un délinquant.

Les préoccupations causalistes, tant quelles sont ignorées, peuvent prendre plusieurs figures, se déguiser, se travestir faisant miroiter à la pensée, autant le chemin que l'hallucination objective de sa trouvaille. Il en va ainsi, dans le site et l'actualité de la «normalité toxicomaniaque».

Après la voie de la légalité, notre écoute s'est en effet frayée une autre voie, celle d'un réalisme naïf et interrogateur à la fois : comment pouvait-il inscrire la toxicomanie du côté de l'anormalité chez les autres et de la normalité chez lui ? Ne reproduisait-il pas, au travers de cette contradiction, cette double tentative de soins, où parallèlement à la «psychothérapie », il suivait un programme de substitution? Et bien d'autres considérations encore, qui s'agrémentaient de figures événementielles et exogènes à l'actualité de la rencontre, faisant chavirer le discours dans le registre de la compréhension, tout ceci sous le couvert du principe de répétition.

Mais la répétition n'était inscrite là que dans le registre de l'écoute... Cause toujours...

Il aura fallu que ce «toxicomane normal» insiste, par un détour associatif, pour libérer notre écoute de ses trépignements. Ce qu'il disait, nous le savions, mais il se produisit un écho nouveau sur notre entendement.

Son père, «psy » à l'étranger, ne consentait toujours pas à lui parler, mais lui avait envoyé un «stylo très cher» au cas où il voudrait lui écrire. «Il a tout fait pour que je m'en sorte, je m'arrêtais mais je rechutais très vite... Je l'ai beaucoup déçu... Il ne me croit plus.»

Notre relation fut ainsi établie de manière à éviter la déception, à défaut de l'analyser. Il aura fallu que ce «père psy », qui ne parlait pas, qui n'entendait pas, qui attendait des écrits, ne se révèle dans et par ce détour transféro-contretransférentiel, pour que notre agitation hypothético-interprétative cesse, là où s'inscrivit pour nous la scansion de cette séance. «La normalité et l'anormalité » du patient avaient, en fait, concentré la substance des mots de cette amnésie infantile, qui n'avait cessée de se diluer dans l'actualité de nos rencontres, via la remémoration dans le langage.

\section{L'exclusion sémantique ou psychologisme, normalisme et autres scientificités...}

La toxicomanie, comme toute autre désignation dans le champ de la psychopathologie à tendance psychiatrique ${ }^{14}$, si elles n'empruntent pas les mêmes chemins diagnostics, tendent pourtant à dériver sur des considérations sciento-causalistes à variables nuancées. Ainsi, par des détours d'explications théoriques sur la (ou les) névrose(s), psychose(s), perversion(s), états-limites, on pourrait fabriquer des 
névrosés, des psychotiques, des pervers et des patients à constitution psychosomatique, et autres «déracinés» ou "déculturés». Tous ces patients fictifs ont la fâcheuse tendance à être en consultation avant même d'avoir pris rendez-vous...

Ces méthodes, à compilations scientistes, compliquent par leurs procédés techniques cette préoccupation causale commune et essentielle de tout homme, nourrissant par là même l'ignorance de ce qu'il sait: incessante quête de sens, vitale pour déterminer la cause de sa destinée. Cette approche dichotomique d'inclusion/exclusion, dedans/dehors, atteint son apogée au travers des sciences cognitivistes qui font de la connaissance mécanique, machiniste, le référentiel du cerveau humain et de son «connexionnisme », là où il suffit de cliquer pour être en contact avec les réseaux sémantiques. On peut alors programmer ou déprogrammer, coder ou décoder, car les systèmes verbaux ne sont reliés entre eux que par des structures, sortes «d'archétypes cognitifs» où la norme est «prototypique».

Si le programme est infecté par un virus - langagier — on procédera à un traitement antiviral pour remettre la programmation neuro-linguistique en marche. Il est alors aisé de localiser les objectifs des thérapies dites cognitivistes. Celles-ci procèdent essentiellement par déplacement, voire par ablation des symptômes comme s'ils ne faisaient pas partie intégrante du sujet humain, de son langage. Elles font appel à un gonflement moïque, véritable réparateur des crevaisons narcissiques par application de rustines, de thérapies visant à normaliser le sujet via les conventions communes.

Cette logique binaire (dedans/dehors, normal/anormal) convoque ici les considérations freudiennes concernant le jugement d'attribution où la «propriété dont il doit être décidé pourrait être originellement avoir été bonne ou mauvaise, utile ou nuisible, exprimée dans le langage des notions pulsionnelles les plus anciennes ». (Freud, 1925, 137) Le principe du normal/anormal, par ses catégorisations et ses généralisations abusives, relève de la «négation» et se présente comme subsistance d'un archaïsme infantile. La causalité qu'il génère ne peut que dévoiler autant qu'elle masque une amnésie tout aussi infantile.

Nous revoilà avec notre patient «toxicomane normal». Que nous propose-t-il dans le site et le gîte de sa parole ? Normal et anormal s'y trouvent en équivalence dans le registre hypothétique de l'inconscient, là où il n'y a pas de contradiction. Ainsi, l'aspiration de l'écoute clinique ne consisterait-elle pas à n'accorder aucune prévalence afin d'autoriser le déploiement de tous les signifiants dans la conjoncture complaisante de leurs sonorités et de leur inscription corporelle et, par là même, permettre au patient d'interroger le choix de ses mots?

Nous postulons que dans ce foyer du langage réside le site de l'interprétation conçu à l'instar de R. Gori (1987), comme une «interpellation» et non sur le registre de «l'extrapolation». Ainsi dans l'actualité des rencontres cliniques, les mots interpellent-ils de leurs effets celui qui écoute. C'est bien de cette cooccurrence trans-subjective que l'interprétation peut naître. Une interprétation qui aurait pour vocation d'ouvrir, non pas tant sur l'un ou l'autre des protagonistes, l'émetteur ou le récepteur, le clinicien ou le patient, que sur le sujet - qui peut 
indistinctement être l'un ou l'autre —, sur cet espace/temps de l'entre-deux que nous nommerons lien transférentiel.

Le savoir sur la toxicomanie à variables psychologiques, sociologiques, anthropologiques, médicales, etc., comme les connaissances théoriques sur d'autres désignations psychopathologiques à causalité neurologique, somatique ou historicopsychique, en tant que dopés par les études, statistiques et autres sériations, viennent, souvent, nourrir les considérations contre-transférentielles dont le leitmotiv est la détermination de la cause par omission de la parole.

Ce rappel vise essentiellement à montrer qu'à l'instar de la toxicomanie, toute dénomination psychopathologique qui œuvre dans les rencontres cliniques à partir de ses théories, verserait dans le déficitaire et «applique ainsi au sujet les limites de sa méthode» (Gori, 1996).

Invoquons-le, le risque est d'autant plus grand pour les cliniciens de verser dans ce paradigme causaliste, au vu du nombre de dispositifs hyper-spécialisés en tout genre dans lesquels ils œuvrent. Notre praxis clinique au sein d'institutions spécialisées, autant auprès de ceux qu'on appelle «toxicomanes» que de ceux qu'on désigne par «migrants », ne cesse de nous confronter à cette mosaïque de théories qui orne les modalités dites de "prise en charge », où la tentation, voire le souci du diagnostic sont constamment présents. Alliance périlleuse du savoir et de la technique, qui livre souvent des systèmes interprétatifs «clés en mains ».

Notre critique ne vise pas pour autant les dispositifs de soins, mais une certaine écoute clinique qui pourrait s'y déployer, oubliant que dispositif ne rime aucunement avec «l'être-là», dans l'actualité de chaque rencontre, sauf à confondre toxicomanie et toxicomane, alcoolisme et alcoolique, précarité et précaire, exclusion et exclu, Rmi et Rmiste, psychose et psychotique... Confusion où phénomènes et sujets tendent à se rejoindre dans une collusion sémantique qui ne pourra plus tenir que par des tautologies, des catégorisations au sens Bachelardien de «généralisations abusives », produisant au mieux par une technicisation de la relation. L'écoute deviendrait ainsi post-toxicomanie, post-exclusion, post-précarité, post-rmi, post-psychose... Écoute affaiblie et déclinée car excluante, exclusive et exclue d'elle-même.

En écho à la logique et à l'éthique analytique, nous proposerons de concevoir ces procédés d'écoute clinique en tant que produits d'impasses contre-transférentielles. À ce titre, ils s'inscrivent dans un "avant-coup" savant; par là même, ils ne seront en mesure que de déployer leurs stratégies propres, à vérifier, et de trouver de manière chimérique les miroitements de leurs fictions hypothético théoriques. Le sujet du langage n'est-il pas alors maintenu au lointain du regard et de l'écoute?

\section{L'heuristique : élan éthique et mouvement «transféro-contre-transférentiel »}

En un mot comme en cent, les psychologues cliniciens ont certes vocation et intérêt heuristique à pratiquer leur métier dans ces dispositifs de plus en plus nombreux, spécialisés et technicisés. Peut-être en s'autorisant à désapprendre, à repenser constamment leurs savoirs, et ce afin d'introduire une souplesse 
conceptuelle en lieu et place des rigidités théoriques et techniques, et de rapatrier les préoccupations causales dans le site du transfert et du contre-transfert. Bref, il s'agirait de faire de la parole cette preuve qui se veut révélation, autant d'une heuristique, que d'une éthique.

L'heuristique s'agence dans un cheminement, qui privilégie la mise en pratique d'une méthode au lieu d'une référence à un système, et où les conceptualisations ne sont que des effets «d'après-coup», dont la prétention universalisable doit laisser ses paradoxes hypothétiques ouverts à d'autres «après-coup». Si l'éthique se conçoit, au sens freudien, comme «limitation aux pulsions» (Freud, 1939), c'est vraisemblablement pour souligner que son assise se dégage de l'analyse du contre-transfert. Ethique et contre-transfert semblent ainsi indissociables dans et d'une pratique clinique.

Ainsi nous ne parlerons pas tant d'éthique de la psychanalyse ou de la psychologie clinique, que d'une éthique du psychanalyste ou du psychologue clinicien. Rappelons ici notre «malaise» contre-transférentiel avec le «toxicomane normal », qui prenait pour fondation les effets des paroles des patients et leur écho dans le roman infantile. Le malaise contre-transférentiel se présenterait alors, pour tout clinicien, dans cette résonance paradoxale, familière et ignorée à la fois. Telle est notre proposition éthique et heuristique majeure, celle, finalement de mettre au défi nos facultés créatrices et inventives pour les remettre en mouvement une fois que la conscience de ce malaise contre-transférentiel aura inscrit ses effets dans le champ de l'oubli et de la remémoration, là où dynamique psychique, économie pulsionnelle et amnésie infantile pourront à nouveau être liées.

À suivre notre démarche, le travail du psychologue clinicien au sein des lieux de soins, spécialisés ou pas, nécessiterait de reconstruire «singulièrement les lieux psychiques de la mémoire personnelle» (Fédida, 1995, 254). Mémoire de la situation clinique permettant la restitution, via le langage, de ces lieux psychiques qui, répétons-le, ne peuvent se construire qu'indépendamment des dispositifs où ils se déploient. À partir d'eux, pourraient jaillir ces paroles/concepts qui sollicitent toute culture de savoir, qu'elle soit théorique ou empirique, pour la soumettre à l'épreuve de la parole.

La cure type, en effet, vaut également par ses variantes. Ces dernières, affectées au champ de l'exclusion, engendrent un mouvement perpétuel ré-créatif et cliniquement re-génératif celui d'inventer et de réinventer chaque fois une nouvelle variante; procédé à même de renouveler un cadre original de rencontre susceptible de par sa spécificité, d'interroger le dispositif dans lequel il se réalise.

La dialectisation clinique et métapsychologique sans fin de l'espace psychique et du dispositif d'accueil, se co-implique afin de se proposer comme topos, lieux psychiques, demeure d'hospitalité de l'Humain et non de l'humanitaire. Par ce cadre il s'agirait de prêter pour un temps son psychisme à l'autre afin qu'il puisse y déposer tout en l'interrogeant la variante de ses demandes telles qu'elles sont adressées à l'institution. C'est cette co-occurrence que nous avons jusqu'alors analysé, et dont nous apporterons quelques témoignages en guise d'ouverture pour cet écrit. 


\section{Pour accompagner le mouvement...}

Notre réflexion qui a pris ici une tonalité épistémo-polémique, a pour origine la confrontation de nos pratiques multiples, tout au long de ses dernières années, aussi bien dans diverses institutions aux prises en charges multiples, associations ou institutions à caractère social, médical ou humanitaire, que dans le champ libéral. Nous œuvrons ainsi, tantôt aux côtés d'autres collègues aux pratiques différentes, et/ou la plus value clinique, si elle vise son opérationnalité, pourrait tendre à être complémentaire et non hégémonique pour ne pas dire écrasante. Tantôt dans la solitude d'une responsabilité privée qui enclin à cheminer exclusivement dans l'«historialité» de chaque rencontre. Le déplacement d'une pratique à l'autre, nous contraint à dresser un certain nombre de constats relatifs au dispositif, à la place et à la gestion du transfert, que nous allons justement en guise d'ouverture, réinterroger de façon dynamique.

Pour ce qui concerne le dispositif, nous remarquerons que celui issu des « variantes de la cure type» (Lacan, 1953), ne peut selon cette analyse, être transposé à l'identique dans d'autres dispositifs à intervenants diversifiés. Les interférences transférentielles qui s'y déploient, dans la multitude des concours et des apports proposés aux patients, imposent de prendre en compte la diversité des offres et demandes, ainsi que le ou les objets qui constituent l'existence, certes essentiellement imaginaire, de l'institution. L'histoire récente est chargée de ces tentatives de conversions propres à ces variantes de la cure type en institution qui, quelques soient leur valeur et/ou leur opérationnalité au sein de leur dispositif, se sont souvent transformées en une application d'un discours analytique qui, en évacuant toute dimension d'altérité sémantique, a fini par exclure et s'exclure. Le clinicien à l'œuvre dans ces champs, ignore ainsi parfois la manière dont chacun des protagonistes, et non moins collègue, investit sa mission. Il lui arrive même de méconnaître la mission de l'autre. L'implication plutôt que l'application, autoriserait à identifier la place de chaque intervenant, par une confrontation éthique et ludique à la pratique de l'autre.

Le propre du symbolique étant d'être confronté à la dialectique de son absence — le mot à la place de la chose —, l'autre en devient susceptible d'accompagnement dans sa mission, au même titre que le clinicien peut-être accompagné dans la sienne. Les savoirs sont alors déconstruits, afin d'inventer des méthodes d'interventions à géographie de signifiants, délimités mais cependant non étanches. L'heuristique qui se dégage de tels processus, dialectise les idéologies, les places et les savoirs, afin d'amener chacun des intervenants à soutenir son dire par une action.

Notre conscientisation d'un besoin des méthodes psychanalytiques en tant qu'impliquées dans le lien social, ne concerne pas uniquement les «psychistes» dans la mesure où cette expérience est la somme de confrontations avec le pathos, qu'il soit individuel, collectif et «collectivisé». Ainsi intéresse-t-elle également les travailleurs sociaux, médicaux, paramédicaux, ayant pour champ d'implication et d'exercice des quartiers dits «défavorisés», et qui s'adressent à des publics précarisés, exclus : actions de prévention, groupes de parole, analyse des pratiques, entretiens à domicile, formation et coordination de femmes-relais médiatrices, lieux 
d'accueil parents-enfants, suivis psychologiques auprès d'un public en situation de précarité sociale, migrants ou non (RMI, API,...). Cette multiplicité d'interventions, que nous ne pouvons complètement énumérer ici ${ }^{15}$, d'accompagnement, de prise en charge se fait conjointement avec les acteurs du travail social. Elle est portée par le concept d' «action/recherche », qui est une démarche participative avec les professionnels et les habitants. Elle vise à donner à chacun une place équivalente au sein des différentes actions menées: celle d'avoir quelque chose à dire. Place équivalente mais non pas égale, le semblable n'étant pas l'identique, car la différence de place entre un professionnel et un usager est absolument irréductible. Elle s'appuie sur la question suivante: "Qu'en disent-ils eux, "eux" qui vivent ou qui travaillent dans les quartiers et dont "on" parle habituellement?»

Ces différentes actions visent les objectifs suivants: comment permettre à chacun de dépasser un enfermement communautaire (ethnique, territorial, professionnel) et/ou une absence de liens, pour s'autoriser quelque chose (une parole, un acte), en son nom propre. Ceci d'ailleurs, comme nous l'avons constaté, autorise chacun à modifier son rapport aux autres personnes du groupe, puisque le travail sur le long terme permet de mettre en évidence le leurre que la dimension groupale constitue.

C'est ainsi que nous avons un jour entendu un éducateur de rue qui nous accompagnait dans nos entretiens à domicile, s'exclamer, non sans humour: «Je faisais n'importe quoi avec les jeunes, je continue à faire n'importe quoi, mais au moins je le sais »; cet Rmiste qui n'était pas venu consulter, mais se faire aider administrativement, et qui, au bout de quelques mois de dépouillement de divers papiers nous dit: «Je ne sais pas si on a arrangé les papiers mais je ne savais pas que j'avais tout ça en moi à dire»; cette femme venue dans le lieu d'accueil parents enfants nous expliquer: «J'accompagne mon fils pour qu'il joue et depuis je parle mieux le français »; ce patient turc, à qui l'assistante sociale, en présence de la psychologue, proposait un stage d'alphabétisation, et à laquelle il répliqua: «Moi, mon alpha, je le fais ici»; enfin, cette stagiaire psychologue, qui à la fin de son expérience d'immersion professionnelle établit le constat suivant: "Maintenant que je sais ce que c'est, je ferai attention à deux fois avant de juger et de reprendre un éducateur sur ce qu'il aurait mal fait...».

jacques cabassut université de nice sophia-antipolis

190, boulevard gambetta 30220 saint-laurent d'aigouze.

mohammed ham université de nice sophia-antipolis/

47 , avenue de l'arrousaire 84000 avignon mohammed.ham@unice.fr 


\section{Notes}

1. Notre écrit est contemporain de la récente ré-exhumation en France, du texte de loi légiférant le champ des psychothérapies (Projet Accoyer), soit la délivrance de son titre et le parcours de formation express en psychologie clinique des futurs psychothérapeutes.

2. Le dictionnaire historique de la langue française nous apprend que le mot exclusion du verbe exclure (de la forme évoluée esclore) provient du latin excludere qui provient de claudere, fermer: enfermer dehors, telle est la dynamique intrinsèque au mouvement exclusif d'exclusion, que le clinicien rapprochera de l'opération de la forclusion. Dans son étymologie, forclore contient en effet l'idée «d'enfermer dehors », le préfixe «for » provient de fores, forium (dehors) et clore de claudo, claudis, claudere (enfermer, fermer). Quant à la Verwerfung freudienne, elle exprime littéralement le fait de «jeter de côté» (Assoun, 1999, 221).

3. Il s'agit de la méthadone, produit de substitution pour les patients notamment héroïnomanes.

4. Nous traiterons ici du double aspect de l'imaginaire: aliénant car narcissiquement défensif/subjectivant et créatif, une fois évidé — sinon nettoyé — d'une partie de sa dimension de rivalité haineuse et spéculaire.

5. Là où le signifiant représente le sujet pour un autre signifiant.

6. Lacan peut définir la haine comme une communion sans communication, nous pourrions affirmer que la recherche d'inclusion à une communauté de signes, quels qu'ils soient, conduirait à la haine. La demande sociale d'inclusion, d'insertion mènerait à ce titre à la même crispation haineuse, à charge pour l'intervenant social de la subvertir. Se reporter à ce propos au «fantasme communionnel» dans son lien au cannibalisme et à l'incorporation, tel que Lacan le développe lors de la séance du 22 mars 1961 du Séminaire sur le transfert. Nous faisons ici référence à un tel fantasme dans l'effet d'inclusion imaginaire (sémantique, communautaire) et d'exclusion symbolique qu'il engendre.

7. Il s'agit d'un néologisme que Lacan crée afin de rendre compte de la double dimension (familière/étrangère) propre à la jouissance.

8. Se reporter à ce sujet au schéma L ou celui du graphe du désir. Lacan, Écrits (1966), p. 53 et 815.

9. Nous faisons référence ici à ces trois temps lacaniens que sont l'instant de voir, le temps de comprendre et le moment de conclure, qui permettent au sujet de cheminer et à l'atemporalité de l'inconscient de se déployer

10. Tel est nous semble t-il le cas du psychologue dans son adresse à l'éducateur dans la séquence de supervision ci avant abordée.

11. C.H.R.S, sleeping, boutiques, points écoutes de centres sociaux, M.J.C, acteurs de quartiers dits sensibles, etc.

12. Nous entendons par là que la captation du sujet par et dans sa propre image, le fixe dans un leurre dont il ne peut que difficilement se dégager.

13. Nous faisons référence à la loi du 31 décembre 1970 apparentant la consommation de drogue à un acte délictueux et répréhensible. Ici comme ailleurs, l'écoute clinique qui agit du côté de l'évènement et qui ne s'interroge pas à partir du site langagier de sa fondation, creuse le trou où s'ensevelissent les mots cadavérisés dés leur naissance, où la sépulture est érigée par les cache-misères des représentations imagées, où les paroles demeurent à jamais endeuillées, et où le rapport à l'autre se noie dans une mélancolie langagière. La primauté de l'événementiel signe l'autodafé de la parole. Elle lui substitue des exogénéítés causalistes pour poursuivre sa quête des images. Ces considérations, quant aux effets des propos du patient sur nous, sont à inscrire dans un après-coup, celui où elles sont revenues avec force là où nous ne les attendions pas, là où l'étonnement et la surprise de leur implication intrapsychique, nous ont entraîné dans des arabesques de mots qui ouvrent sur d'autres mots.

14. Psychopathologique, psychanalytico-structuraliste, psychosomatique, ethnopsychiatrique, opérant à partir de phénomènes environnementaux, neurologiques, somatiques ou somato-psychiques...

15. Le descriptif et l'analyse clinique et métapsychologique de ces actions recherches, menées essentiellement par des psychologues cliniciens et des femmes relais médiatrices, se trouvent dans les bilans d'activités de Migrations Santé Vaucluse : 25/27 rue Bonneterie-84000 Avignon. 
Filigrane, automne 2007

\section{Références}

Assoun, P-L., 1999, Le préjudice et l'idéal, pour une clinique sociale du trauma, Paris, Anthropos.

Derrida, J., 1999, Sur parole, Paris, Éditions de L’Aube

Fedida, P., 1992, Crise et contre-transfert, Paris, P.U.F., Coll. Psychopathologies.

Fedida, P., 1995, Le site de l'étranger, Paris, P.U.F.

Freud, S., 1915, L'inconscient, Métapsychologie, Paris, Gallimard, Folio-Essais, 1968, 65-121.

Freud, S., 1925. La négation, Résultats, idées, problèmes II, 1921-1938, Paris, P.U.F, Tr. Fr. 1985, 135-139.

Freud, S., 1939, L’homme Moïse et la religion monothéiste, Paris, Gallimard, Folio-essais, Tr. Fr. 1986.

Gori, R., 1996, La preuve par la parole, sur la causalité en psychanalyse, Paris, P.U.F, Coll. Psychopathologie.

Lacan, J., 1953, Variantes de la cure-type, in Écrits, Paris, Seuil, 1966, 323-362.

Lacan, J., 1960-61, Le Séminaire, Livre VIII, Le transfert, Paris, Seuil, 1991.

Lesourd, S. 2006, Comment taire le sujet? Des discours aux parlottes libérales, Ramonville Saint Agne, érès Editions. Legendre, P., 2001, De la société comme texte. Linéaments d'une anthropologie dogmatique, Paris, Fayard.

Legendre, P., 2004, Ce que l'Occident ne voit pas de l'Occident. Conférences au Japon, Paris, Arthème-Fayard, Mille et une nuits, Coll. Les quarante piliers.

Pujol, R., 1977, «Des noms qui causent», Comment l'interprétation vient au psychanalyste, Paris, Aubier.

Pujol, R., 1992, «La scène primitive : à revoir », Nouvelle Revue de psychanalyse, 46, 25-39. 